SPIN

Vol. 7, No. 1 (2017) 1740001 (10 pages)

(C) World Scientific Publishing Company

DOI: 10.1142/S201032471740001X

\title{
CFA Films in Amorphous Substrate: Structural Phase Induction and Magnetization Dynamics
}

\author{
M. A. Correa* and F. Bohn \\ Departamento de Física, Universidade Federal do Rio Grande do Norte \\ 59078-900 Natal, RN, Brazil \\ *marciocorrea@dfte.ufrn.br \\ V. M. Escobar \\ Departamento de Física, Universidade Federal de Santa Maria \\ 97105-900 Santa Maria, RS, Brazil \\ Received 14 December 2016 \\ Accepted 14 March 2017 \\ Published 25 May 2017
}

\begin{abstract}
We report a systematic study of the structural and quasi-static magnetic properties, as well as of the dynamic magnetic response through $\mathrm{MI}$ effect, in $\mathrm{Co}_{2} \mathrm{FeAl}$ and $\mathrm{MgO} / / \mathrm{Co}_{2} \mathrm{FeAl}$ single layers and a $\mathrm{MgO} / / \mathrm{Co}_{2} \mathrm{FeAl} / \mathrm{Ag} / \mathrm{Co}_{2} \mathrm{FeAl}$ trilayered film, all grown onto an amorphous substrate. We present a new route to induce the crystalline structure in the $\mathrm{Co}_{2} \mathrm{FeAl}$ alloy and verify that changes in the structural phase of this material leads to remarkable modifications of the magnetic anisotropy and, consequently, dynamic magnetic behavior. Considering the electrical and magnetic properties of the $\mathrm{Co}_{2} \mathrm{FeAl}$, our results open new possibilities for technological applications of this full-Heusler alloy in rigid and flexible spintronic devices.
\end{abstract}

Keywords: Heusler material; magnetization dynamics; magnetoimpedance.

\section{Introduction}

The broad range of phenomena in the fields of spintronics and magnonics with potential for technological applications requires the development and optimization of ferromagnetic materials with specific static and dynamic magnetic properties. While systems with high damping parameter are explored in spin pumping and inverse spin Hall effect applications, ${ }^{1-3}$ systems with low damping parameter present a remarkable importance for magnonics devices ${ }^{4-6}$ and ultrafast sensors. ${ }^{7,8}$

Regarding low damping materials, the FullHeusler materials ${ }^{9}$ composed by $\mathrm{Co}_{2} \mathrm{FeAl}$ (CFA) corresponds to an interesting alternative for injection and detection of spin-polarized currents, ${ }^{10}$ even though a completely disordered structure is observed. ${ }^{11}$ In particular, this alloy, besides the low damping parameter, can reach up to $100 \%$ spin polarized current at the Fermi level, justifying the strong interest on this material in the last years. At the same time, the static magnetic characteristics of CFA can be employed in distinct devices based on/or exploring the induced uniaxial anisotropy and/or magnetoelastic properties. ${ }^{12}$

With respect to the magnetic properties of CFA full-Heusler alloy, a complex magnetic behavior has been previously reported. ${ }^{13}$ Thus, the exploration of

${ }^{*}$ Corresponding author. 
its applicability requires investigations of the magnetization dynamics in saturated and nonsaturated magnetic states of the samples, as well as, in resonant and nonresonant regimes. To this end, the Magnetoimpedance (MI) effect presents itself as an interesting tool. The MI effect corresponds to the changes in the electrical impedance of a ferromagnetic material when submitted to an external magnetic field. The MI response is strongly dependent on the magnetic anisotropy and its orientation with the applied fields in the experiment, ${ }^{14-18}$ bringing insights on the relations among structural and magnetic properties and magnetization dynamics.

The production of samples with controlled structural, electrical and magnetic properties is a hard task. The full-Heusler alloys can be described by $\mathrm{X}_{2} \mathrm{YZ}$, where $\mathrm{X}$ and $\mathrm{Y}$ are transition metals, as $\mathrm{Co}, \mathrm{Fe}$ and $\mathrm{Ni}$, and $\mathrm{Z}$ is an element of the sp group, e.g., $\mathrm{Al}$ and $\mathrm{Si}$. The well-known crystallographic structures L2 $2_{1}, \mathrm{~B} 2$ and A2, associated to the type of occupation of the three available lattice sites, are strongly dependent on the growth parameters and subsequent annealing procedures. ${ }^{10}$ The obtainment of the aforementioned crystallographic structural phases are usually performed by growing the layers onto $\mathrm{MgO}$ or $\mathrm{Si}$ oriented substrates. ${ }^{7,19}$ Although these substrates are rigid, this obtainment is an important step to the development of spintronic devices in flexible organic substrates, in which the employed substrate usually presents amorphous structure.

Motivated by these features, in this paper we present a new route to induce crystalline structure in the CFA alloy grown onto amorphous substrate. We explore the structural and quasi-static magnetic properties, as well as the dynamic magnetic response through MI effect, in CFA single layers and a trilayered film. We show that the structural phase of the CFA alloy onto amorphous substrate evolutes from an amorphous state to an A2 crystalline phase by the insertion of a $\mathrm{MgO}$ buffer layer. This change in the structural phase leads to remarkable modifications of the magnetic anisotropy and, consequently, dynamic magnetic behavior. Since the main features of the films in glass amorphous substrates can be mirrored in nanostructures grown onto flexible substrates, ${ }^{20,21}$ our results correspond to an important step to the employment of the CFA alloy in flexible spintronic devices.

\section{Experimental Procedure}

For this study, we produce films with three different geometries. The first one is a $\mathrm{Co}_{2} \mathrm{FeAl}(500 \mathrm{~nm})$ single layer. For the other films, we consider a $5 \mathrm{~nm}$ thick $\mathrm{MgO}$ buffer layer and grow a $\mathrm{MgO} / / \mathrm{Co}_{2} \mathrm{FeAl}$ $(500 \mathrm{~nm})$ single layer and a $\mathrm{MgO} / / \mathrm{Co}_{2} \mathrm{FeAl}$ $(250 \mathrm{~nm}) / \mathrm{Ag}(100 \mathrm{~nm}) / \mathrm{Co}_{2} \mathrm{FeAl}(250 \mathrm{~nm})$ trilayered film. Figures $1(\mathrm{a})-1(\mathrm{c})$ presents a schematic representation of the produced films. The samples are grown onto amorphous glass substrates with dimensions of $6 \times 6 \mathrm{~mm}^{2}$. They are deposited by the magnetron sputtering technique using the following parameters: base pressure of $6 \times 10^{-7}$ Torr, Ar pressure during disposition of $10^{-3}$ Torr, $90 \mathrm{~W}$ set in the RF source for the deposition of the $\mathrm{MgO}$ layer, and $100 \mathrm{~W}$ and $10 \mathrm{~W}$ in a DC source for the CFA and Ag layers, respectively. Using these parameters, the deposition rates are $0.02 \mathrm{~nm} / \mathrm{s}, 0.56 \mathrm{~nm} / \mathrm{s}$ and $0.18 \mathrm{~nm} / \mathrm{s}$ for $\mathrm{MgO}, \mathrm{CFA}$ and $\mathrm{Ag}$, respectively. To induce magnetic anisotropy, an external magnetic field of 100 Oe is applied in the plane of the substrate during the deposition process. In particular, for the production, the substrate is previously annealed at $450^{\circ} \mathrm{C}$ for $1 \mathrm{~h}$. The $\mathrm{MgO}$ and $\mathrm{Ag}$ layers are deposited at room temperature, while the CFA layers are grown with the substrate at $150^{\circ} \mathrm{C}$. The employed temperature is chosen based on a systematic study of the structural and magnetic properties for epitaxial CFA films performed by Qiao et al. ${ }^{22}$

The samples are characterized from the structural and magnetic point of views. The structural

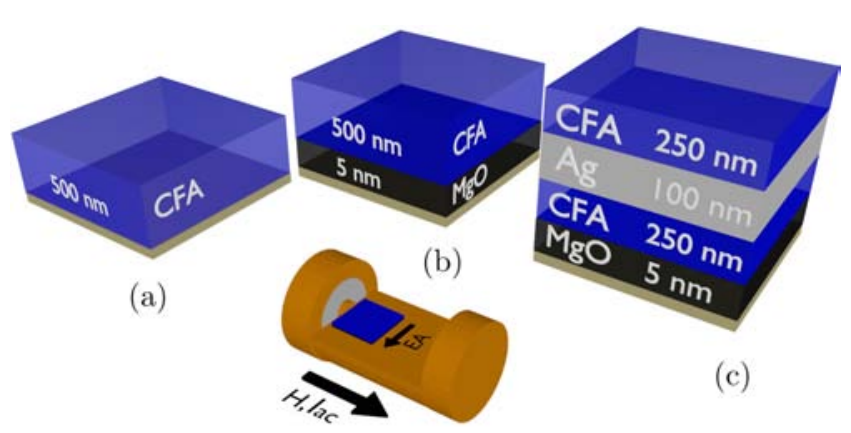

(d)

Fig. 1. (a)-(c) Schematic representation of the produced films. Amorphous glass substrates are employed for the production of all studied samples. (d) Microstrip in which the sample is the central conductor. Here we show the transverse MI configuration, in which the easy magnetization axis (EA) is oriented transverse to the external magnetic field $H$ and the electrical current $I_{a c}$. 
CFA Films in Amorphous Substrate

characterization is obtained through X-ray diffraction (XRD), experiments performed using a Rigaku MiniFlex system. The quasi-static magnetic properties are analyzed via magnetization curves, acquired with a LakeShore Model 7407 vibrating sample magnetometer, with maximum external inplane magnetic field of \pm 350 Oe.

Finally, the magnetization dynamics is investigated through MI measurements. The MI experiments are performed using an impedance analyser Agilent model E4991, with E4991A test head connected to a stripline, i.e., a microstrip in which the sample is the central conductor, as shown in Fig. 1(d). The real $R$ and imaginary $X$ components of the impedance $Z$ are measured over a wide range of frequencies, from $0.3 \mathrm{GHz}$ up to $3.0 \mathrm{GHz}$, in a linear regime with $1 \mathrm{~mW}(0 \mathrm{dBm})$ constant power, and magnetic field varying between \pm 350 Oe. The measurements are acquired with the field in the plane of the films in two configurations: (i) Longitudinal MI, in which the probe current and external magnetic field are applied along the easy magnetization axis of the sample and (ii) transverse MI, where both are applied transverse to the easy axis. In particular, these MI configurations differentiate by rotating the sample, i.e., modifying the easy axis with respect to the $H$ and $I_{a c}$ direction.

\section{Results and Discussions}

Here, we present and discuss the results related to the structural and magnetic characterization, as well as to the MI response of the films.

Figure 2 shows the XRD results for CFA films. For the CFA single layer deposited onto glass substrate without the $\mathrm{MgO}$ buffer layer, an amorphous structural character is evidenced. On the other hand, the films for grown onto the $\mathrm{MgO}$ buffer layer present the CFA [022] texture, assigned by the welldefined and high intensity peak at $44.73^{\circ}$. Moreover, the trilayered film, besides the $\mathrm{Ag}$ [111] texture, also shows the CFA [004] for one, identified by the presence of a small peak located at $65.12^{\circ}$.

Considering the CFA structural phases, the absence of [111] and [002] textures allows us to infer that, except for the sample without the $\mathrm{MgO}$ layer, the CFA alloy presents the A2 structure, since the [022] and [422] textures are present, associated with the complete chemical disorder among the $\mathrm{Co}, \mathrm{Fe}$ and $\mathrm{Al}$ sites. ${ }^{10,13,23}$ Due the thicknesses of the CFA and $\mathrm{Ag}$ layers, no information on the $\mathrm{MgO}$ layer is

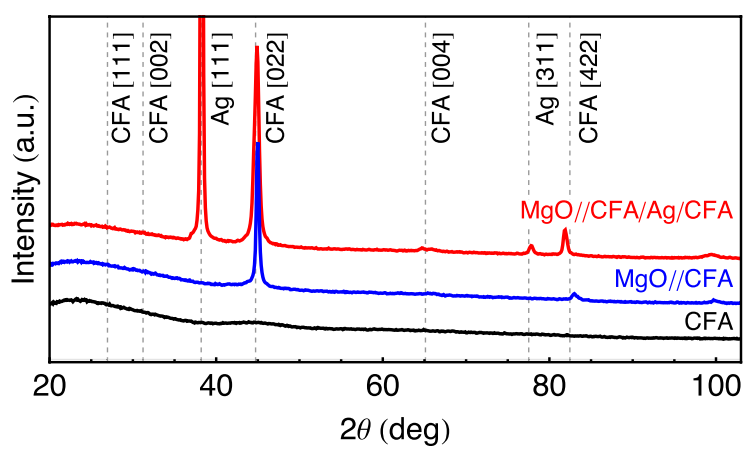

Fig. 2. XRD results for the CFA single layer, the $\mathrm{MgO} / / \mathrm{CFA}$ single layer, and $\mathrm{MgO} / / \mathrm{CFA} / \mathrm{Ag} / \mathrm{CFA}$ trilayered film. The dashed vertical lines indicate the patterns obtained from ICDD 01-071-5670 and ICDD 03-065-2871 for CFA and Ag, respectively. In particular, the peaks associated to the CFA alloy indicate the A2 disordered phase.

obtained. However, the insertion of this $\mathrm{MgO}$ buffer is responsible for CFA structural orientation, given that the sample produced without the $\mathrm{MgO}$ layer presents amorphous structural character.

The magnetic properties of the CFA alloy are strongly dependent on the growth process. Thus, similar to the structural character, the magnetic anisotropy presents a dependence with sample geometry. Figures 3(a)-3(c) show normalized magnetization curves obtained for the CFA films. For the CFA single layer, despite of a magnetic field is applied during the deposition, isotropic in-plane magnetic properties are verfied. This behavior can be associated with the stress stored during the deposition and with the low structural quality of this sample. Similar features have been previously reported for a $\mathrm{CFA} / \mathrm{Au} / \mathrm{CFA}$ trilayered film grown onto amorphous glass substrate. ${ }^{11}$ For the CFA single layer, a small coercive field of $H_{c} \approx 2.0 \mathrm{Oe}$ is found for both measurements, while the field saturation $H_{s}$ is around $150 \mathrm{Oe}$, not shown here.

On the other hand, with the insertion of the $\mathrm{MgO}$ buffer layer, the quasi-static magnetic behavior presents noticeable changes. The flatness of the $\mathrm{MgO}$ layer over the substrate enables the growth of samples with structural phase induction. Thus, the magnetic field applied during the deposition, combined with the structural order and quality in the interface, gives rise to the magnetic anisotropy in the CFA layers.

For the $\mathrm{MgO} / / \mathrm{CFA}$ single layer, the angular dependence of the curves indicates an uniaxial in-plane magnetic anisotropy, induced by the field applied during the deposition. The easy magnetization axis is 


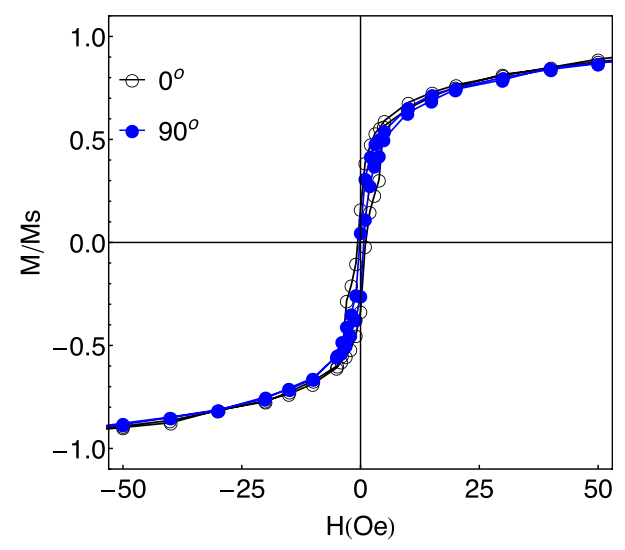

(a)

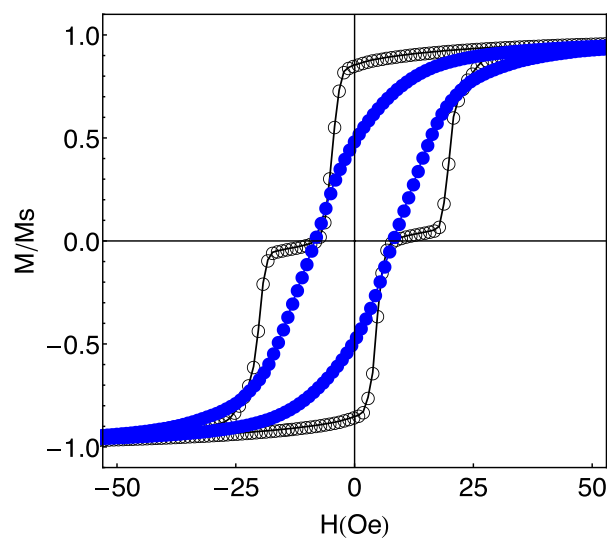

(c)

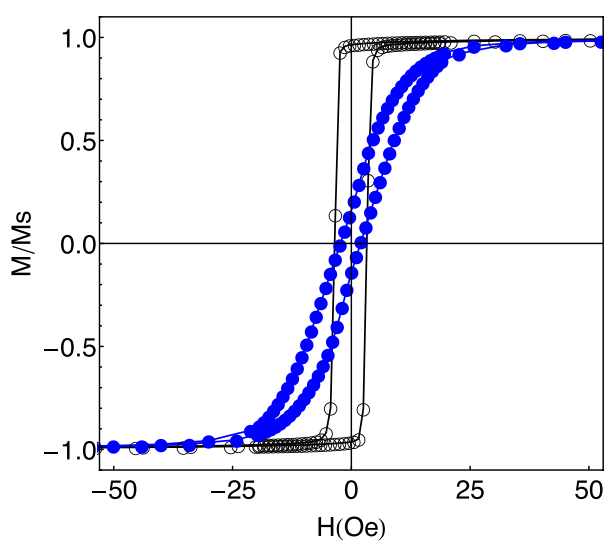

(b)

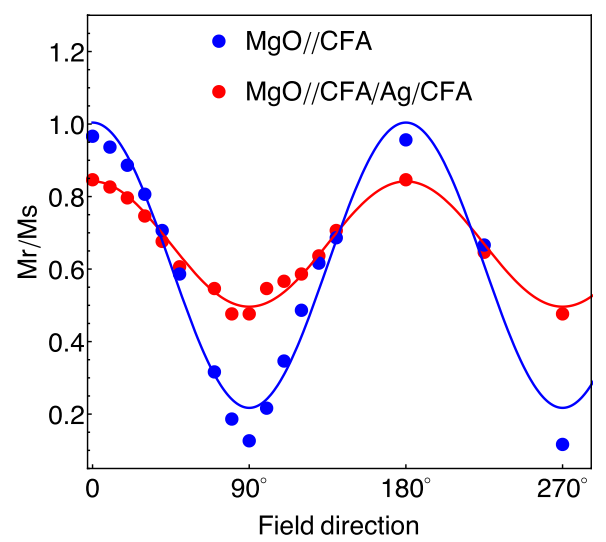

(d)

Fig. 3. Normalized magnetization curves obtained for the (a) CFA single layer, (b) $\mathrm{MgO} / / \mathrm{CFA}$ single layer and (c) $\mathrm{MgO} / / \mathrm{CFA} /$ $\mathrm{Ag} / \mathrm{CFA}$ trilayered film. The curves are acquired with external field along $\left(0^{\circ}\right)$ and perpendicular $\left(90^{\circ}\right)$ to the direction of the magnetic field applied during the deposition. (d) Normalized remanent magnetization as a function of external magnetic field direction for the $\mathrm{MgO} / / \mathrm{CFA}$ single layer and $\mathrm{MgO} / / \mathrm{CFA} / \mathrm{Ag} / \mathrm{CFA}$ trilayered film. The trends of the experimental results reveal the uniaxial anisotropy direction.

identified in the measurement at $0^{\circ}$, for which presents a squared curve with coercive field of $H_{c} \approx 5.0$ Oe. For the measurement at $90^{\circ}$, a slightly tilted loop with smaller remanence appears once the magnetic field is perpendicular to the easy axis. In this case the saturation field is $H_{s} \approx 25 \mathrm{Oe}$.

For the $\mathrm{MgO} / / \mathrm{CFA} / \mathrm{Ag} / \mathrm{CFA}$ trilayered film, a biphase magnetic behavior is found. The two-stage magnetization process is characterized by the magnetization reversal of one CFA layer at low fields, followed by the reversal of the other CFA layer at higher fields. In principle, the biphase magnetic behavior suggests that the CFA layers are uncoupled. The easy magnetization axis remains along the direction of the field applied in the substrate during the deposition, as expected. Thus, this magnetic behavior can be associated with distinct magnetic properties of CFA layers. The CFA layer grown onto $\mathrm{MgO}$ buffer layer keeps the soft magnetic properties, with switching field of around 5.0 Oe for the measurement at $0^{\circ}$, while the other one grown onto the $\mathrm{Ag}$ layer presents harder magnetic properties, with switching field of $\approx 22$ Oe. This fact reflects the striking influence of the seed layer in which the CFA is deposited. The plateau located at around zero magnetization indicate an antiparallel alignment between the magnetization of the CFA layers. This is a fingerprint of the same magnetic contribution of both layers, and similar induced magnetic anisotropy direction of the CFA layer, despite they have distinct anisotropy constants.

For both samples produced using the $\mathrm{MgO}$ buffer layer, uniaxial anisotropy is confirmed by the behavior of the $M_{r} / M_{s}$ as a function of the applied 
field direction, as shown Fig. 3(d). The maxima in the trend curves indicate the easy magnetization direction, while the minima indicate the hard axis one. From a general point of view, the trilayered film presents higher anisotropy induction, when compared with CFA film grown onto $\mathrm{MgO}$ buffer layer. It is related with the stress stored during deposition and its dependence with the CFA layer thicknesses.

It is well-known that quasi-static magnetic properties play a fundamental role in the dynamic magnetic response and MI behavior. ${ }^{24,25}$ The shape and amplitude of the MI curves are dependent on the orientation of the applied field and $a c$ current with respect to the magnetic anisotropies, magnitude of the external field and probe current frequency as well as are directly related to the mechanisms responsible for the transverse magnetic permeability changes: skin and ferromagnetic resonance (FMR) effects. In particular, at moderate frequency range $(\mathrm{MHz})$, the skin depth is smaller than $t / 2$, where $t$ is the thickness of the sample. This mechanism changes the transverse permeability of the sample and, consequently, the MI. At high frequency range $(\mathrm{GHz})$, beside the strong skin effect, the FMR effect is present in the MI measurement, changing strongly the transverse permeability. ${ }^{26,27}$ Based on that, experiments of longitudinal and transverse MI are investigated. Given that the CFA single layer has isotropic magnetic anisotropy, here just the results of the anisotropic $\mathrm{MgO} / / \mathrm{CFA}$ single layer and $\mathrm{MgO} / / \mathrm{CFA} / \mathrm{Ag} / \mathrm{CFA}$ trilayered film are explored.

Using the microstrip configuration, where the sample is the central conductor, both electrical and magnetic contributions are obtained during the MI measurement. To make easier a direct comparison between the results obtained through the longitudinal and transverse MI measurements, the normalized $Z$ is variation is considered. To this end, $\Delta R / R$ and $\Delta X / X$ are calculated through

$$
\Delta R / R=\frac{R(H)-R\left(H_{\max }\right)}{R\left(H_{\max }\right)},
$$

and

$$
\Delta X / X=\frac{X(H)-X\left(H_{\max }\right)}{X\left(H_{\max }\right)},
$$

where $R(H)$ and $X(H)$ are the real and imaginary components of the impedance at a given external magnetic field $(H)$, and $R\left(H_{\max }\right)$ and $X\left(H_{\max }\right)$ are the respective components at $H=350 \mathrm{Oe}$, the maximum value of the external magnetic field, where the samples are in the magnetic saturation state. This procedure is performed for each one of the frequencies employed in the experiment.

Figure 4 presents the $\Delta R / R$ and $\Delta X / X$ normalized components of the impedance as a function of the external magnetic field for the $\mathrm{MgO} / / \mathrm{CFA}$ single layer for the frequency range of $0.5 \mathrm{GHz}$ up to $3.0 \mathrm{GHz}$, for the $\mathrm{MgO} / / \mathrm{CFA}$ single layer. Although the curves are acquired over a complete magnetization loop and present hysteretic behavior, here just part of the curve, when the field goes from the maximum positive value to maximum negative one, is presented. For the transverse MI experiment [Figs. 4(a) and 4(b)], where the external magnetic field and electrical ac current are applied perpendicularly to the easy magnetization axis [see Fig. 1(d)], the curves present a double peak structure for the whole frequency range, as expected, ${ }^{13,16}$ since the $a c$ field generating by the $I_{a c}$ current is parallel to the easy magnetization axis. At the intermediate frequency range, the $R$ peaks position is close to the $H_{s}$ values verified through the magnetization curves. On the other hand, as the frequency is raised, the $R$ and $X$ peaks displace toward higher fields, a behavior verified above $\sim 2.0 \mathrm{GHz}$ which is related to the increase of the FMR effect contribution to the MI variations. ${ }^{18}$

For the longitudinal MI experiment [Figs. 4(c) and 4(d)], a clear change of the peaks structure with frequency is found, as expected. In this configuration, the easy magnetization axis is along the external magnetic field and electrical current. Consequently, the $R$ component of the impedance presents a single peak structure at the intermediate frequency range. As the frequency is raised, the FMR effect becomes mainly responsible for the MI variations, splitting the single peak in a double peak structure at $\sim 2.2 \mathrm{GHz}$ [see horizontal dashed line in Fig. 4(c)]. Above this value, as the frequency is increased, there is the wellknown displacement of the peaks toward higher fields as the frequency is increased.

These results reveal the dependence of the dynamic magnetic response with the probe current frequency and magnetic field. In this sense, it is possible to explore another representation of the same data by using the plot of the imaginary component as a function of the real one. ${ }^{3,28}$ Figure 5 shows the $\Delta X / X$ as a function of $\Delta R / R$ for selected frequencies, for the $\mathrm{MgO} / / \mathrm{CFA}$ single layer. At this frequency range, below $1.4 \mathrm{GHz}$, we have identified 

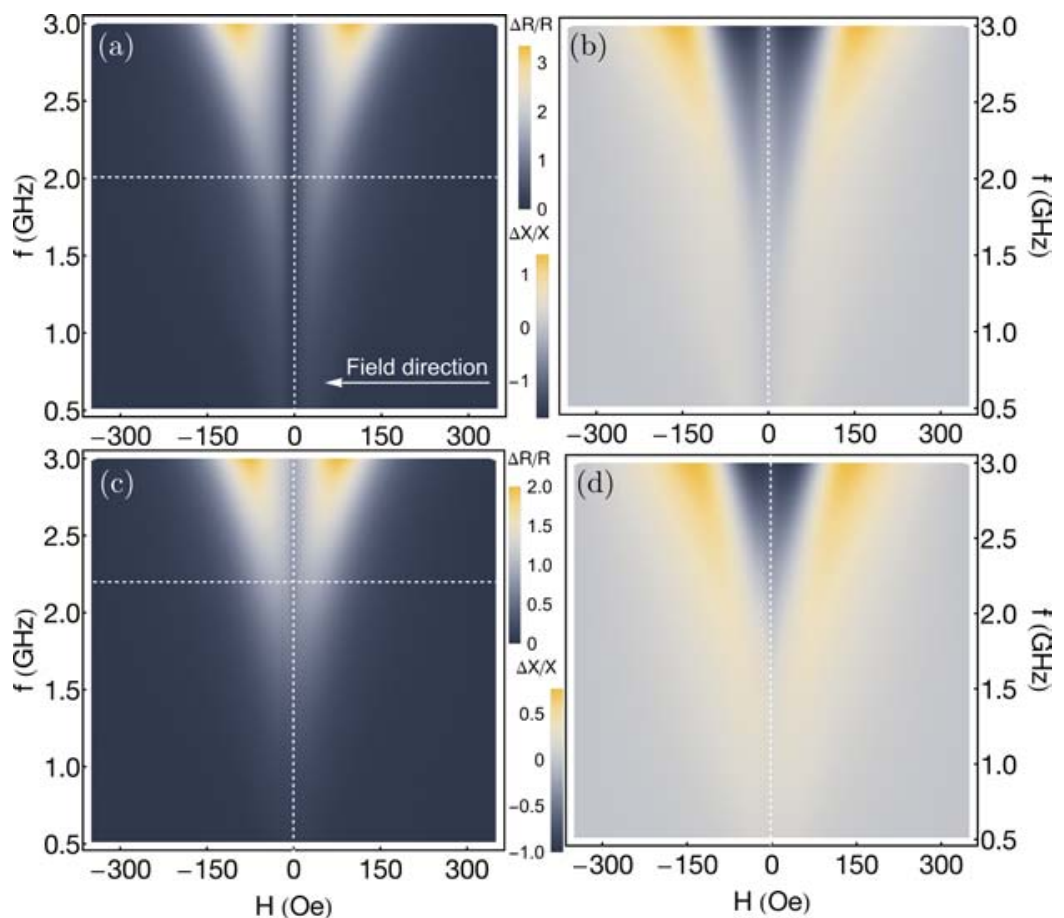

Fig. 4. (a) $\Delta R / R$ and (b) $\Delta X / X$ components of the impedance as a function of the external magnetic field, for the frequency range of $0.5 \mathrm{GHz}$ up to $3.0 \mathrm{GHz}$, for the $\mathrm{MgO} / / \mathrm{CFA}$ single layer in the transverse MI experiment. (c, d) Similar plot for the longitudinal MI experiment. Although the curves are acquired over a complete magnetization loop and present hysteretic behavior, here just part of the curve, when the field goes from positive to negative values, are presented. This fact is represented by the arrow in (a). The horizontal dashed lines in (a) and (c) indicate the frequency values in which the FMR effect becomes the main mechanism responsible for the MI variations.

that the strong skin effect changes the transverse magnetic permeability and, consequently, the MI variations. However, this analysis allows us to verify the frequency limit where the FMR effect starts acting in the sample and contributing for the MI

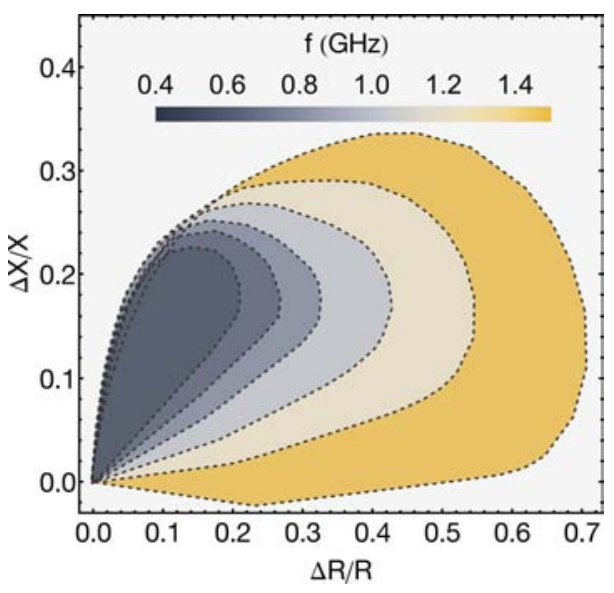

(a) effect. For both MI experiments, the FMR contribution is verified even for the lowest employed frequencies, $\sim 0.4 \mathrm{GHz}$. It can be found from the fact that the experimental points follow a circular trajectory. ${ }^{28,29}$ This behavior is more evident in

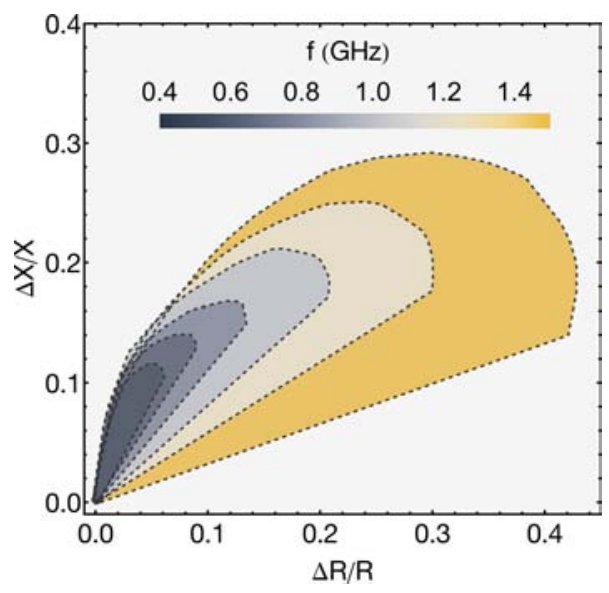

(b)

Fig. 5. $\Delta X / X$ component as a function of the $\Delta R / R$ one for selected frequencies for the (a) transverse and (b) longitudinal MI experiments for the $\mathrm{MgO} / / \mathrm{CFA}$ single layer. In particular, notice that even at low frequencies, the FMR effect contributes to the MI variations. 
transverse MI experiment [Fig. 5(a)], since the alternating magnetic field $H_{a c}$ is along the easy magnetization axis, triggering the excitation of local magnetic anisotropies.
Similar analyses are performed for the $\mathrm{MgO} / /$ $\mathrm{CFA} / \mathrm{Ag} / \mathrm{CFA}$ trilayered film. They are respectively presented in Figs. 6 and 7. The insertion of the metallic Ag layer leads to a reduction of the

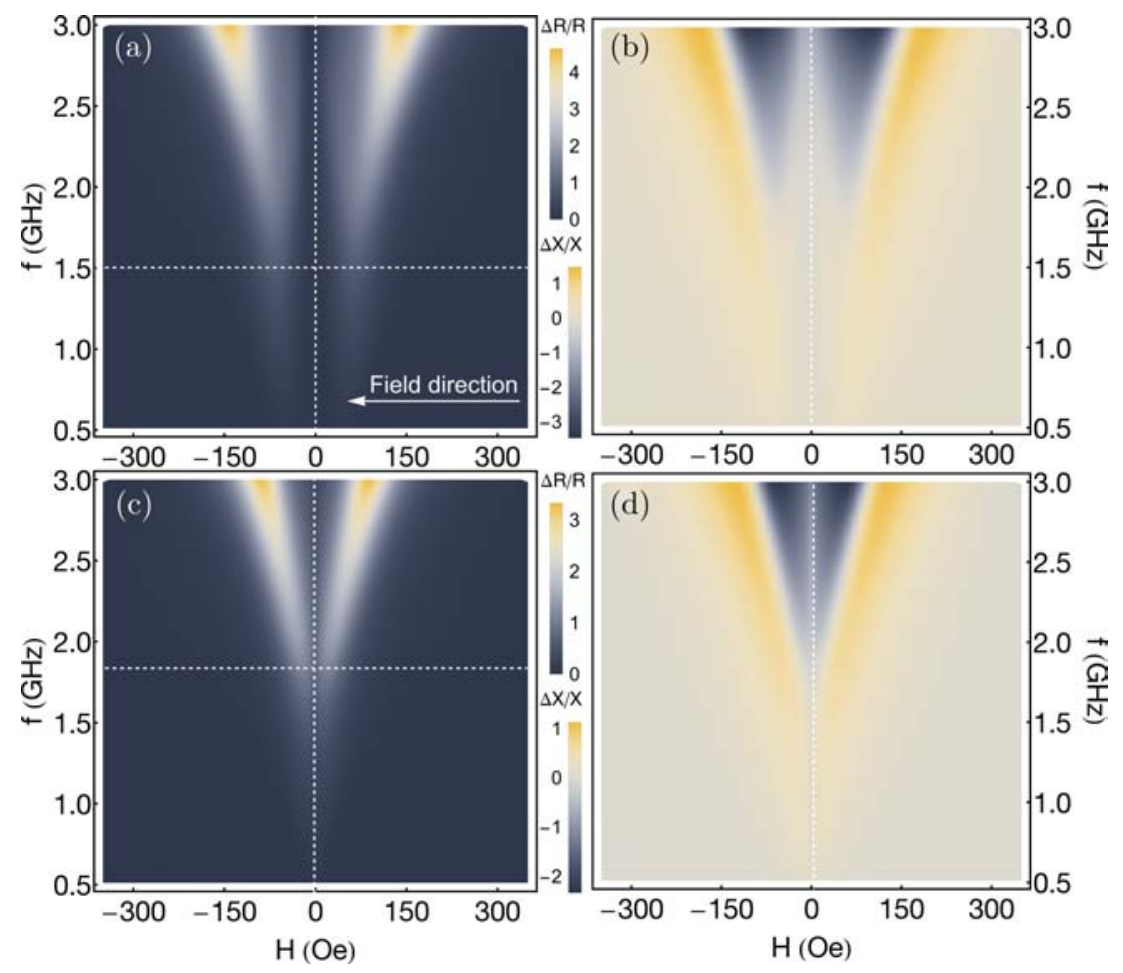

Fig. 6. (a) $\Delta R / R$ and (b) $\Delta X / X$ components of the impedance as a function of the external magnetic field, for the frequency range of $0.5 \mathrm{GHz}$ up to $3.0 \mathrm{GHz}$, for the $\mathrm{MgO} / / \mathrm{CFA} / \mathrm{Ag} / \mathrm{CFA}$ trilayered film in the transverse MI experiment. (c, d) Similar plot for the longitudinal MI experiment. Although the curves are acquired over a complete magnetization loop and present hysteretic behavior, here just part of the curve, when the field goes from positive to negative values, are presented. This fact is represented by the arrow in (a). The horizontal dashed lines in (a) and (c) indicate the frequency values in which the FMR effect becomes the main mechanism responsible for the MI variations.

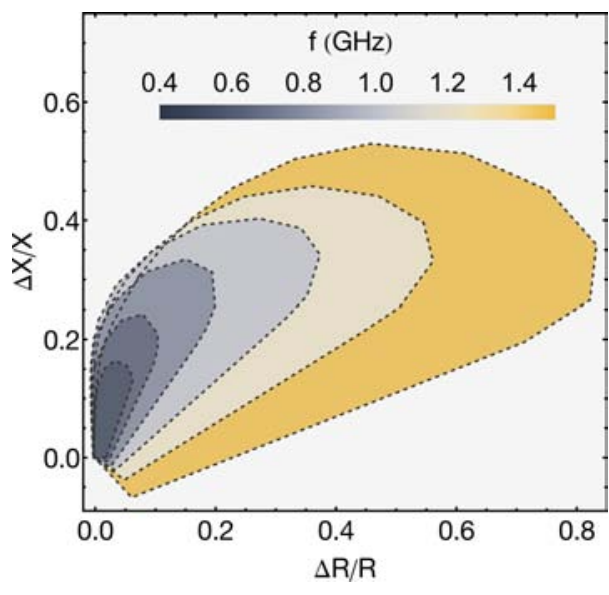

(a)

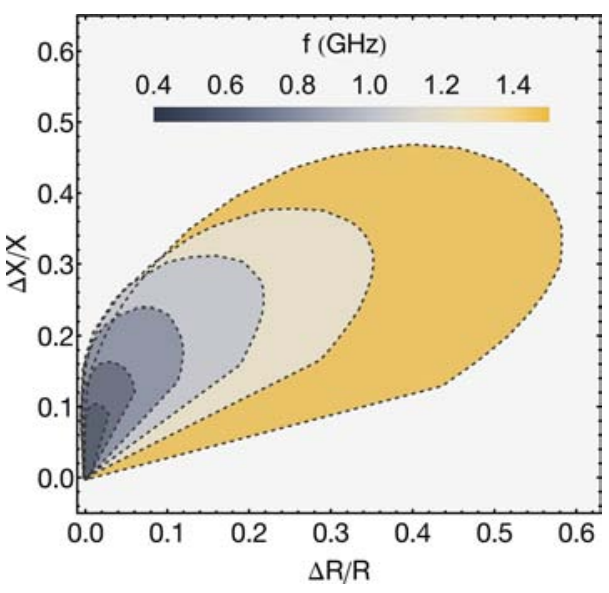

(b)

Fig. 7. $\Delta X / X$ component as a function of the $\Delta R / R$ one for selected frequencies at the (a) transverse and (b) longitudinal MI experiments for the $\mathrm{MgO} / / \mathrm{CFA} / \mathrm{Ag} / \mathrm{CFA}$ trilayered film. In particular, notice that even at low frequencies, the FMR effect contributes to the MI variations. 
electrical resistance of the whole sample. This feature improves the MI performance even the magnetic volume of the sample does not change. Although the behavior related to the peaks structure and mechanisms governing the magnetization dynamics are similar, for the trilayered film noticeable characteristics are revealed. As expected, the quasi-static magnetic properties are reflected in the magnetization dynamics. At the intermediate frequency range, the peaks are located at $\sim 45$ Oe in the transverse MI experiment [Fig. 6(a)]. At the same time, the frequency limits where the FMR effect becomes the main mechanism responsible for the MI variations decrease considerably, when compared with the value found for the sample without the Ag layer. In this case, for the transverse MI experiment, the frequency is $\sim 1.5 \mathrm{GHz}$, while for the longitudinal MI one it is $\sim 1.8 \mathrm{GHz}$.

The limits between the frequency ranges in which distinct mechanisms act in the MI variations are related with the magnetic anisotropy of the films. These magnetic features can be observed in the plot of the $\Delta X / X$ as a function of $\Delta R / R$ (Fig. 7). In this sample, the FMR effect contributes, irrespective of MI experiment (transverse or longitudinal), with the MI variation from $0.4 \mathrm{GHz}$. However, for both, the circular trajectories are not completely well defined, a fact related to the imperfect alignment between alternating magnetic field and magnetic anisotropy, due to anisotropy dispersion, different than that observed in Fig. 5(a).

Finally, Fig. 8 shows the $\Delta R / R$ versus $H$ at 0.4 $\mathrm{GHz}$ for the $\mathrm{MgO} / / \mathrm{CFA}$ single layer and $\mathrm{MgO} / /$ $\mathrm{CFA} / \mathrm{Ag} / \mathrm{CFA}$ trilayered film, together with magnetization curves, reflecting the relation between the quasi-static magnetic properties and dynamic magnetic response. For both, the results represent the classical MI behavior for an anisotropic system when the electrical current and the external magnetic field are applied transverse to the easy axis direction. In particular, the peaks are located close to the $H_{s}$ values found from the magnetization curves. Besides, for the trilayered film, a plateau near zero field is observed (see the red rectangle in the Fig. 8(b)), reflecting the quasi-static magnetic properties for this sample. Despite the biphase magnetic behavior, no evidence of asymmetric MI effect is verified. This is a striking signature of the uncoupling of the ferromagnetic CFA layers in the trilayered film. ${ }^{25}$

From a general point of view, the features found for the CFA films may be of interest for a wide sort of future applications. The manipulation of thicknesses, spacers, buffer and seed layers in these geometries enables us to taylor the dynamic magnetic response and to tune the frequemcy limits in which the FMR effect emerges. At the same time, the insertion of the $\mathrm{MgO}$ buffer layer allows us to obtain crystalline CFA films with very interesting magnetic

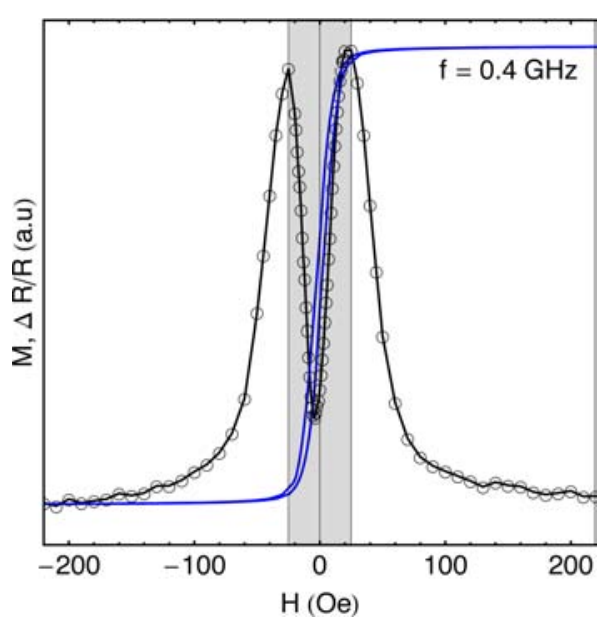

(a)

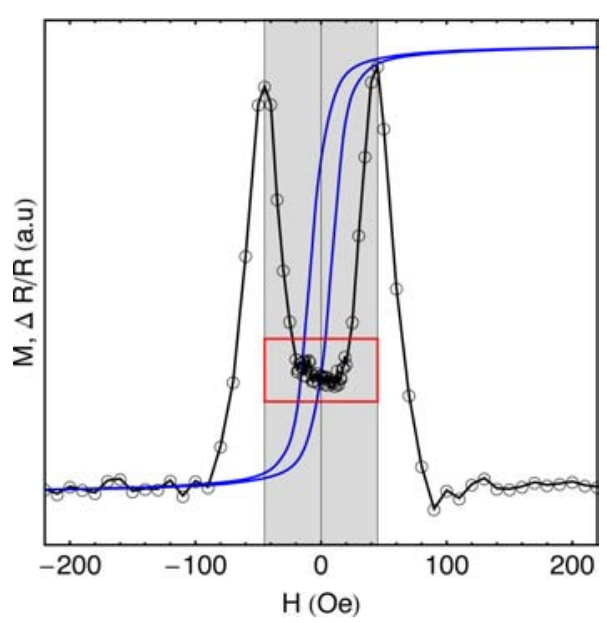

(b)

Fig. 8. $\Delta R / R$ versus $H$ curves in the transverse $\mathrm{MI}$ experiment at the low frequency range, where the skin effect is the main mechanism responsible by the $\mathrm{MI}$ variations, and magnetization curve at $90^{\circ}$ for the (a) $\mathrm{MgO} / / \mathrm{CFA}$ single layer and (b) $\mathrm{MgO} / /$ $\mathrm{CFA} / \mathrm{Ag} / \mathrm{CFA}$ trilayered film. In (a), notice the correspondence of the $\Delta R / R$ peak position and the $H_{s}$ in the magnetization curve. On the other side, in (b), observe the plateau in the $\Delta R / R$ curve, highlighted by the red rectangle, a fact reflecting the quasi-static magnetic properties for this sample (color online). 
properties and rich magnetization dynamics. The integration of the present study with future works, in which the properties of the CFA alloy are explored in flexible amorphous substrates, is an utmost question for the employment of the CFA alloys in flexible spintronics devices.

\section{Conclusions}

In conclusion, we have investigated the structural features, quasi-static magnetic properties, and dynamic magnetic response through magnetoimpedance effect of $\mathrm{Co}_{2} \mathrm{FeAl}$ and $\mathrm{MgO} / / \mathrm{Co}_{2} \mathrm{FeAl}$ single layers and of a $\mathrm{MgO} / / \mathrm{Co}_{2} \mathrm{FeAl} / \mathrm{Ag} / \mathrm{Co}_{2} \mathrm{FeAl}$ trilayered film, all grown onto amorphous substrate. By considering a $\mathrm{MgO}$ buffer layer, we have induced changes in the structural phase of the alloy, thus showing a new route to induce crystalline structure in the $\mathrm{Co}_{2} \mathrm{FeAl}$ alloy. The amorphous $\mathrm{Co}_{2} \mathrm{FeAl}$ single layer has isotropic in-plane magnetic properties. However, the $\mathrm{MgO} / / \mathrm{Co}_{2} \mathrm{FeAl}$ single layer has presented the well-known magnetic behavior verified in anisotropic systems with uniaxial magnetic anisotropy. Moreover, the $\mathrm{MgO} / / \mathrm{Co}_{2} \mathrm{FeAl} / \mathrm{Ag} / \mathrm{Co}_{2} \mathrm{FeAl}$ trilayered film has revealed a biphase magnetic behavior, a feature previously verified in systems as $\mathrm{NiFe} /$ spacer/Co nanostructures. Thus, the evolution from an amorphous to an A2 crystalline structure has led to remarkable modifications of the magnetic anisotropy and, consequently, in the magnetic dynamic response. In this sense, we have played with the structural phase, magnetic anisotropy and dynamic magnetic response of CFA films. The fact that the main features of films in glass amorphous substrates can be mirrored in films grown onto flexible substrates places our results as an important step to the employment of the CFA alloy in flexible spintronic devices. We hope that our investigations motivate experimentalists to the development of this scientific story.

\section{References}

1. Z. Duan, C. T. Boone, X. Cheng and I. N. Krivorotov, Phys. Rev. B 90, 24427 (2014).

2. A. Ruiz-Calaforra, T. Bracher, V. Lauer, P. Pirro, B. Heinz, M. Geilen, A. V. Chumak, A. Conca, B. Leven and B. Hillebrands, J. Appl. Phys. 117, 163901 (2015).

3. M. A. Corrêa, R. Dutra, T. L. Marcondes, T. J. A. Mori, F. Bohn and R. L. Sommer, Mater. Sci. Eng. B Solid-State Mater. Adv. Technol. 211, 115 (2016).
4. M. A. W. Schoen, D. Thonig, M. L. Schneider, T. J. Silva, H. T. Nembach, O. Eriksson, O. Karis and J. M. Shaw, Nat. Phys. 12, 839 (2016).

5. D. Houssameddine, U. Ebels, B. Delaët, B. Rodmacq, I. Firastrau, F. Ponthenier, M. Brunet, C. Thirion, J.-P. Michel, L. Prejbeanu-Buda, M.-C. Cyrille, O. Redon and B. Dieny, Nat. Mater. 6, 441 (2007).

6. H. Ulrichs, B. Lenk and M. Münzenberg, Appl. Phys. Lett. 97, 092506 (2010).

7. M. Belmeguenai, H. Tuzcuoglu, M. S. Gabor, T. Petrisor, C. Tiusan, F. Zighem, S. M. Chérif and P. Moch, J. Appl. Phys. 115, 43918 (2014).

8. G. Ortiz, M. S. Gabor, T. Petrisor Jr., F. Boust, F. Issac, C. Tiusan, M. Hehn and J. F. Bobo, J. Appl. Phys. 109, 07D324 (2011).

9. S. Mizukami, D. Watanabe, M. Oogane, Y. Ando, Y. Miura, M. Shirai and T. Miyazaki, J. Appl. Phys. 105, 07 D306 (2009).

10. M. Belmeguenai, M. S. Gabor, F. Zighem, Y. Roussigné, D. Faurie and C. Tiusan, Phys. Rev. B 94, 104424 (2016).

11. M. A. Corrêa, V. M. Escobar, O. Trigueiro-Neto, F. Bohn, K. D. Sossmeier, C. G. Bezerra, C. Chesman, J. Pearson and A. Hoffmann, Appl. Phys. Express 6, 93001 (2013).

12. M. Gueye, B. M. Wague, F. Zighem, M. Belmeguenai, M. S. Gabor, T. Petrisor, C. Tiusan, S. Mercone and D. Faurie, Appl. Phys. Lett. 105, 62409 (2014).

13. A. M. Silva, V. M. Escobar, G. L. Callegari, K. Agra, C. Chesman, F. Bohn and M. A. Corrêa, Mater. Let. 156, 90 (2015).

14. L. Kraus, J. Magn. Magn. Mater. 195, 764 (1999).

15. L. Kraus, Sens. Actuators A Phys. 106, 187 (2003).

16. R. L. Sommer and C. L. Chien, Appl. Phys. Lett. 67, 857 (1995).

17. R. L. Sommer and C. L. Chien, Appl. Phys. Lett. 67, 3346 (1995).

18. A. Yelon, D. Menard, M. Britel, P. Ciureanu, D. Ménard, M. Britel and P. Ciureanu, Appl. Phys. Lett. 69, 3084 (1996).

19. M. Belmeguenai, H. Tuzcuoglu, M. S. Gabor, T. Petrisor, C. Tiusan, D. Berling, F. Zighem, T. Chauveau, S. M. Chérif and P. Moch, Phys. Rev. B 87, 184431 (2013).

20. K. Agra, T. Mori, L. S. Dorneles, V. M. Escobar, U. C. Silva, C. Chesman, F. Bohn and M. Corrêa, J. Magn. Magn. Mater. 355, 136 (2014).

21. K. Agra, F. Bohn, T. J. A. Mori, G. L. Callegari, L. S. Dorneles and M. A. Correa, J. Magn. Magn. Mater. 420, 81 (2016).

22. S. Qiao, S. Nie, J. Zhao and X. Zhang, J. Appl. Phys. 113, 233914 (2013).

23. J. M. De Teresa, D. Serrate, R. Cordoba and S. M. Yusuf, J. Alloy. Compd. 450, 31 (2008). 
24. R. B. da Silva, M. A. Corrêa, E. F. Silva, T. J. A. Mori, R. D. D. Pace, R. Dutra, A. D. C. Viegas, F. Bohn and R. L. Sommer, Appl. Phys. Lett. 104, 102401 (2014).

25. E. F. Silva, M. Gamino, A. M. H. De Andrade, M. A. Corrêa, M. Vázquez and F. Bohn, Appl. Phys. Lett. 105, 102409 (2014).

26. M. A. Corěra, F. Bohn, V. M. Escobar, M. S. Marques, A. D. C. Viegas, L. F. Schelp and R. L. Sommer, J. Appl. Phys. 110, 93914 (2011).
27. M. A. Corrêa, F. Bohn, R. B. da Silva and R. L. Sommer, J. Appl. Phys. 116, 243904 (2014).

28. J. M. Barandiaran, A. Garcia-Arribas and D. de Cos, J. Appl. Phys. 99, 103904 (2006).

29. M. A. Correa, F. Bohn, C. Chesman, R. B. da Silva, A. D. C. Viegas and R. L. Sommer, J. Phys. D. Appl. Phys. 43, 295004 (2010). 